\title{
Editorial Ein Blick zurück nach vorn
}

Wissenschaftliche Zeitschriften, wenn sie lange genug erscheinen, setzen Jahresringe an, dickere, dünnere, gelegentlich unregelmäßige. Der Jahrgang 2003 unserer Zeitschrift wird sich, wenn künftige Leser auf die Gesamtentwicklung schauen, als ein auffälliger Jahresring abzeichnen. Communicatio Socialis hat 35 Jahre hinter sich, nach gängiger Menschenleben-Zählung mehr als eine Generation. In dieser Zeit wurde die Zeitschrift kontinuierlich von ihren Gründern herausgegeben: von FranzJosef Eilers, dem Vater der Idee und dem Initiator und Motor der Verwirklichung im Jahr 1968, sowie von Michael Schmolke und Karl Höller, den Mitherausgebern seit der ersten Stunde.

Diese Gründer sind seither wie die Zeitschrift selbst 35 Jahre älter geworden. Es war an der Zeit, Ausschau zu halten nach Nachfolgern, die das Fortbestehen im Sinne der Ursprungsidee sichern können. Zugleich galt es wieder einmal, die materielle Existenz zu garantieren. Das gelingt bei geistes- und sozialwissenschaftlichen Zeitschriften nur mit Hilfe von Förderern; denn allein aus dem Verkaufserlös trägt sich das Unternehmen nicht, und der Anzeigenerlös hat nur ideellen Wert.

Beides ist im vergangenen Jahr gelungen: die Herausgeberschaft $\mathrm{zu}$ verjüngen und das weitere Erscheinen der Zeitschrift sicherzustellen. Aus dem bisherigen Triumvirat wird Michael Schmolke auch in Zukunft zur Verfügung stehen. An die Stelle von Franz-Josef Eilers und Karl Höller treten Walter Hömberg vom Lehrstuhl für Journalistik der Katholischen Universität Eichstätt-Ingolstadt und Matthias Kopp vom Bereich Kirche und Gesellschaft im Sekretariat der Deutschen Bischofskonferenz. Fester Ankerplatz unserer Zeitschrift ist also ab jetzt die Katholische Universität Eichstätt-Ingolstadt. Sie tritt somit auch organisatorisch in die inhaltlich nahe liegende Verbindung zu einem renommierten Journalistik-Studiengang und damit zur Kommunikationswissenschaft im weiteren Sinne.

Die Herausgeber fühlen sich dem Programm des Gründungsjahres verpflichtet. Im ersten Heft schrieb Franz-Josef Eilers unter der Überschrift „Publizistik als Aufgabe“: „Die neue Zeitschrift soll solchen Erkenntnissen und Erfahrungen aus dem Gesamtbereich der Publizistik ihre Seiten öffnen, die mittelbar oder unmittelbar für kirchliche publizistische Arbeit bedeutsam sein können. Sie soll durch Beiträge von Fachleuten auf Probleme und Aufgaben hinweisen und zu ihrer Lösung beitragen - durch 
Information, Anregung und Kritik. Communicatio Socialis wird dann aber auch versuchen, nach und nach einen Überblick über die publizistischen Aktivitäten der Christen in aller Welt zu geben, um dadurch zu neuen Initiativen anzuregen oder bei begonnenen Arbeiten Mut zu machen."

Zuvor hatte zum ersten $\mathrm{Mal}$ in der Geschichte ein Konzil grundlegend zur Kommunikation der Gesellschaft Stellung genommen (Dekret Inter mirifica von 1963). Noch gab es keine Pastoralinstruktion, die unserer Arbeit dann eine ganz konkrete Aufgabe gestellt hat (Communio et progressio 1971). Aber „die Auseinandersetzung mit den publizistischen Mitteln" war damit, wie Eilers schrieb, „eine Aufgabe geworden, die nicht mehr mit schiefen Blicken oder dem erhobenen Zeigefinger begleitet werden soll. Jeder allerdings, der sich dieser Tatsache stellt, merkt bald, wie vielfältig und vielschichtig der Gesamtbereich der Publizistik im allgemeinen und einzelner publizistischer Disziplinen im besonderen inzwischen schon geworden ist."

Der Gesamtbereich der öffentlichen gesellschaftlichen Kommunikation ist seither noch vielfältiger und vielschichtiger geworden. Man denke nur an die Einführung und Durchsetzung dualer Rundfunksysteme und an die grundstürzende Veränderung aller Informations- und Kommunikationsvorgänge auf der Basis der elektronischen Digitalisierung, für die als Stichworte nur PC und Internet stehen mögen.

Verändert hat sich auch die Sicht auf die Aufgabenstellung des Zweiten Vatikanischen Konzils. Manche der damals in den Vordergrund gerückten Schwerpunkte werden nicht mehr als so dringend angesehen. Die Nutzung der so wunderbaren Kommunikationsmittel durch kirchliche Institutionen scheint von der strategischen wieder auf die taktische Ebene zurückgedrängt zu werden.

Communicatio Socialis ist ihrer Aufgabe, die neue, aus dem Konzil hervorgegangene Betrachtungsweise öffentlicher Kommunikation zu begleiten und $\mathrm{zu}$ vertreten, stets treu geblieben. Interdisziplinarität und Internationalität - diese Ziele werden wir weiterhin im Visier haben. Im Übrigen wollten wir stärker als in den letzten Jahren zu aktuellen Debatten beitragen und auf Kontroversen eingehen. Auch der Transfer zur journalistischen Praxis und zu weiteren Praxisfeldern der gesellschaftlichen Kommunikation ist eine Aufgabe, der wir uns neu stellen wollen. Hier bleiben Brückenbauer gesucht.

Im Jahre 1968 (!) hat P. Franz-Josef Eilers SVD unsere Zeitschrift - im wörtlichen Sinne - erfunden. Er hat das Konzept entwickelt und den 
(damals hochaktuellen) Titel vorgeschlagen: „Communicatio Socialis“ ist der offiziellen Überschrift des meist nach seinen Anfangswörtern „Inter mirifica“ zitierten Konzilsdekrets vom 4. Dezember 1963 entlehnt; sie lautet "Decretum de instrumentis communicationis socialis". Er hat die Unterstützung seines Ordens für das Projekt gefunden und zwei Mitarbeiter gewonnen: Michael Schmolke und, wenig später, Karl Höller.

Seither hat Franz-Josef Eilers die Zeitschrift mit Umsicht und großem Einsatz geleitet. Als er von seinem Orden auf die Philippinen berufen wurde, blieb er nicht nur ein fleißiger Beiträger, sondern auch Ratgeber in schwierigen Fällen, kurzum der Patron der Zeitschrift. Seine Mitherausgeber und seine Nachfolger sind ihm zu tiefem Dank verpflichtet ebenso wie die gesamte "scientific community" des Arbeitsfeldes Religionskommunikation und die zahlreichen Praktiker, die von seinen Beiträgen über Dritte-Welt-Themen und Missionspublizistik profitiert haben.

Der Theologie-Professor Helmuth Rolfes, der unsere Zeitschrift zwölf Jahre lang redaktionell geleitet hat, wird uns - zum Bedauern der Herausgeber - auf dem neuen Wegesabschnitt nicht weiter begleiten. Er hat sich um die Ausweitung des Themenspektrums große Verdienste erworben. Ohne die Gewinnung immer neuer - nicht nur kompetenter, sondem zum Teil auch prominenter - Autoren wäre das nicht möglich gewesen. Auf diesem Feld lagen, abgesehen von der verantwortungsbewussten Betreuung der üblichen Redaktionsagenden, seine größten Verdienste. Auch als Autor hat er der Zeitschrift in der Überschneidungszone zwischen Theologie und Kommunikationswissenschaft Gewicht verliehen. Die Herausgeber danken ihm herzlich dafür.

Die Redaktion übernimmt dankenswerterweise zunächst Ferdinand Oertel. Er ist mit dem Themenfeld insgesamt, den Strukturen der Kirchen und ihrer Organisationen seit vielen Jahren ebenso vertraut wie mit dem Handwerk des Redakteurs. Im Redaktionsteam von Communicatio Socialis hat er bereits seit 1991 mitgearbeitet.

Wissenschaftliche Zeitschriften sind heute ganz allgemein in einer bedrängten Situation. Ihr Publikumsreservoir ist, besonders im Falle einer thematischen Spezialisierung, begrenzt. Die neuen Herausgeber sehen das nicht als Anlass zur Resignation, sondern als Herausforderung. Sie wissen, dass Communicatio Socialis viel genutzt wird und stellen sich zuversichtlich der Aufgabe, die Zeitschrift fortzuführen als Plattform der wissenschaftlichen Diskussion über Kommunikation in Religion, Kirche und Gesellschaft.

Michael Schmolke - Walter Hömberg - Matthias Kopp 